\title{
Observation of Splintered Josephson Vortices at Grain Boundaries in $\mathrm{YBa}_{2} \mathrm{Cu}_{3} \mathrm{O}_{7-\delta}$
}

\author{
R. G. Mints and Ilya Papiashvili \\ School of Physics and Astronomy, Raymond and Beverly Sackler Faculty of Exact Sciences, Tel Aviv University, Tel Aviv 69978, Israel \\ J. R. Kirtley \\ IBM Thomas J. Watson Research Center, P.O. Box 218, Yorktown Heights, New York 10598 \\ H. Hilgenkamp \\ Low Temperature Division, Department of Applied Physics and MESA ${ }^{+}$Research Institute, University of Twente, P.O. Box 217, \\ 7500 Enschede, The Netherlands \\ G. Hammerl and J. Mannhart \\ Experimentalphysik VI, Center for Electronic Correlations and Magnetism, Institute of Physics, Augsburg University, \\ D-86135 Augsburg, Germany \\ (Received 18 April 2002; published 23 July 2002)
}

\begin{abstract}
We have directly observed well-separated Josephson vortex splinters with unquantized magnetic flux at asymmetric $45^{\circ}$ grain boundaries in $\mathrm{YBa}_{2} \mathrm{Cu}_{3} \mathrm{O}_{7-\delta}$ films by imaging magnetic flux with scanning SQUID microscopy. The existence of these splinter vortices has been predicted and is well described by a model based on $d_{x^{2}-y^{2}}$ pairing symmetry and facetting of the grain boundary on a length scale shorter than the Josephson penetration depth.
\end{abstract}

DOI: 10.1103/PhysRevLett.89.067004

PACS numbers: $74.60 . \mathrm{Ge}, 74.50 .+\mathrm{r}$

The rigid phase coherence of the superconducting state results in quantization of fluxoids and generally also of magnetic flux penetrating superconductors. This is the case for Abrikosov vortices in type-II superconductors [1] and Josephson vortices in Josephson junctions [2]. Exploiting the $d$-wave symmetry of high- $T_{c}$ superconductors, in tricrystalline samples half magnetic flux quanta have been observed in recent years [3]. In contrast to these structures containing precisely quantized flux, completely unquantized flux has been found to be generated by superconducting grain boundaries in high- $T_{c}$ superconductors [4] due to the $d$-wave symmetry and facetting of the grain boundary plane.

In this Letter, we report the existence of a novel type of magnetic flux structure in superconductors. Using scanning SQUID microscopy we have imaged the flux generated by $45^{\circ}$ grain boundaries in superconducting $\mathrm{YBa}_{2} \mathrm{Cu}_{3} \mathrm{O}_{7-\delta}$ films. While magnetic flux of these structures is unquantized, we find that several of them form pairs with magnetic fluxes that sum to $\phi_{0}$ and therefore present splintered vortices.

The asymmetric $45^{\circ}$ [001]-tilt grain boundaries in $\mathrm{YBa}_{2} \mathrm{Cu}_{3} \mathrm{O}_{7-\delta}$ films have two remarkable anomalous behaviors. First, the Josephson critical current dependence on the applied magnetic field $H$ has a non-Fraunhofer characteristic [5-8], with almost no central peak at $H=0$ and possibly with two dominant side peaks at a certain field $H_{m} \neq 0$. Second, unquantized flux spontaneously generated by these grain boundaries is observed upon cooling in zero field [4]. These two fundamental observations have no explanation within a standard model treating a grain boundary as a conventional Josephson junction. A consis- tent understanding has been achieved by combining two specific additional facts [4].

The first fact is the existence of a $d_{x^{2}-y^{2}}$ wave symmetry component of the superconducting order parameter in $\mathrm{YBa}_{2} \mathrm{Cu}_{3} \mathrm{O}_{7-\delta}[3,9]$. This $d_{x^{2}-y^{2}}$ wave symmetry introduces a strong dependence of the Josephson critical current density on the misorientation angle between adjacent grains. The second fact is the facetted structure of the grain boundaries in $\mathrm{YBa}_{2} \mathrm{Cu}_{3} \mathrm{O}_{7-\delta}$ films, with facets having all length scales below $100 \mathrm{~nm}[4,10-14]$. This grain boundary meandering leads to variation of the microscopic grain boundary plane. In some cases these variations are highly important, e.g., when the critical current density $j_{c}$ alternates along the asymmetric $45^{\circ}$ [001]-tilt grain boundary with a nearly zero average value $\left\langle j_{c}(x)\right\rangle$, where $x$ axis is along the macroscopic (averaged over the facets' length scale) grain boundary plane [8].

A high-resolution scanning SQUID microscope $[15,16]$ was used to image the local magnetic fields. With this technique nine samples were studied at $4.2 \mathrm{~K}$, of which we present in this Letter three data series: $\mathcal{A}$ (shown in Ref. [4]) and $\mathcal{B}$ taken from one sample in two different cooldowns, and $C$ taken from another sample (new data). In particular, we report the experimental observation of spontaneously generated splinter vortices with a wide variety of fluxes at the asymmetric $45^{\circ}$ [001]-tilt grain boundaries in $\mathrm{YBa}_{2} \mathrm{Cu}_{3} \mathrm{O}_{7-\delta}$ films.

The samples used for the experiment are [001] $\pm 1^{\circ}$ oriented $\mathrm{YBa}_{2} \mathrm{Cu}_{3} \mathrm{O}_{7-\delta}$ films grown by standard pulsedlaser deposition on bicrystalline $\mathrm{SrTiO}_{3}$ substrates to a thickness of $\simeq 150 \mathrm{~nm}$. The low- $T_{c}$ SQUIDs used for these measurements had an octagonal integrated shielded 
superconducting pickup loop $4 \mu \mathrm{m}$ in diam; at each step of the measurements the pickup loop was shifted by one half of its width (for details, see Ref. [4]).

The alternating critical current density approach has been successfully used to consider Josephson properties of asymmetric grain boundaries in $\mathrm{YBa}_{2} \mathrm{Cu}_{3} \mathrm{O}_{7-\delta}$ films [17-19]. The analytical calculations and numerical simulations demonstrate the existence of spontaneously generated unquantized magnetic flux if (a) the amplitude of alternations of $j_{c}(x)$ exceeds a threshold value, and (b) the length of the facets $l$ is less than the local Josephson penetration depth $\lambda_{\text {loc }}=\sqrt{c \phi_{0} / 16 \pi^{2} \lambda\left\langle\left|j_{c}(x)\right|\right\rangle}$ (here $\lambda$ is the London penetration depth). This flux oscillates with a length scale of $l$ and an amplitude less than the flux quantum $\phi_{0}$.

Another important result follows from the alternating critical current density model [18]. It is predicted that the spontaneous tunnel current loops generate Josephson vortices with unquantized magnetic flux $\phi<\phi_{0}$ (splinter vortices) and perplexing properties: if $j_{c}(x)$ is periodic, then there are two types of splinter vortices carrying fluxes $\phi_{1} \leq \phi_{0} / 2$ and $\phi_{2} \geq \phi_{0} / 2$, related by a complementarity condition $\phi_{1}+\phi_{2}=\phi_{0}$.

The approach to search for the splintered vortices is based on the results of analytical and numerical studies of the electromagnetic properties of the tunnel junctions with an alternating critical current density $[18,19]$. It was shown in these studies that the phase difference generated by a single vortex splinter alternates around certain average values at the vortex "tails." The amplitude of the alternations is small; the average values are from the two series $\varphi_{n}^{+}=2 \pi n+\psi_{\gamma}$ and $\varphi_{n}^{-}=2 \pi n-\psi_{\gamma}$, where $n$ is an integer and the parameter $\psi_{\gamma} \leq \pi / 2$. The series $\varphi_{n}^{+}$and $\varphi_{n}^{-}$interchange being separated by the two "steps" $\Delta_{1}=$ $\varphi_{n}^{+}-\varphi_{n}^{-}=2 \psi_{\gamma}$ and $\Delta_{2}=\varphi_{n+1}^{-}-\varphi_{n}^{+}=2 \pi-2 \psi_{\gamma}$. The fluxes $\phi_{1}$ and $\phi_{2}$ carried by splinter vortices are given by $\phi_{1}=\phi_{0} \Delta_{1} / 2 \pi=\phi_{0} \psi_{\gamma} / \pi$ and $\phi_{2}=\phi_{0} \Delta_{2} / 2 \pi=$ $\phi_{0}-\phi_{1}$, the values of $\phi_{1} \leq \phi_{0} / 2$ and $\phi_{2} \geq \phi_{0} / 2$ are related by the complementarity condition $\phi_{1}+\phi_{2}=\phi_{0}$.

It is important to note that the steps $\Delta_{1}$ and $\Delta_{2}$ are determined at the tails of the splinter vortices. If these steps do not vary along a grain boundary, then the complementarity relation is "global," i.e., it holds for $\phi_{1}$ and $\phi_{2}$ measured for vortices at any location. If $\Delta_{1}$ and $\Delta_{2}$ vary along a grain boundary, then $\phi_{1}$ and $\phi_{2}$ depend on the location of the vortices and the complementarity relation is "local," i.e., it holds only if $\phi_{1}$ and $\phi_{2}$ are measured for closely located vortices.

Consider now a phase pattern with a "dilute" row of splinter vortices, such that the distance between these vortices exceeds the Josephson penetration depth $\Lambda=$ $\sqrt{c \phi_{0} / 16 \pi^{2} \lambda\left\langle j_{c}(x)\right\rangle}$ defined by the averaged value of the critical current density $\left\langle j_{c}(x)\right\rangle \ll\left\langle\left|j_{c}(x)\right|\right\rangle$ [19]. Assume the vortex row starts with slight variations of the phase difference $\varphi(x)$ around $\langle\varphi(x)\rangle=\psi_{\gamma}$. Thus, $\psi_{\gamma}$ is the value of $\langle\varphi(x)\rangle$ at the tail of the neighboring vortex or antivortex. If this neighbor is a vortex, then it has the flux $\phi_{2}$ and $\langle\varphi(x)\rangle$ increases from $\psi_{\gamma}$ to $2 \pi-\psi_{\gamma}$ in this neighbor's domain. If the neighbor is an antivortex, then it has the flux $-\phi_{1}$ and $\langle\varphi(x)\rangle$ should decrease from $\psi_{\gamma}$ to $-\psi_{\gamma}$. This reasoning allows for the understanding of any dilute row of splinter vortices.

This understanding of flux patterns in junctions with an alternating critical current density will now be used to analyze the experimental data on the unquantized magnetic flux localized at the asymmetric $45^{\circ}$ [001]-tilt grain boundaries in $\mathrm{YBa}_{2} \mathrm{Cu}_{3} \mathrm{O}_{7-\delta}$ films. To reveal the splinter vortices we calculate the phase difference $\varphi(x)$ across a grain boundary using the data on the flux $f(x, y)$ measured by the pickup coil of a SQUID microscope (the $y$ axis is assumed perpendicular to the macroscopic grain boundary). First, we find the magnetic flux per step

$$
\phi(x)=\int_{y_{-}}^{y_{+}} f(x, y) \frac{d y}{A},
$$

where $y_{+}$is above and $y_{-}$is below the grain boundary. The values of $y_{+}$and $y_{-}$are defined so that $f\left(x, y_{+}\right)$and $f\left(x, y_{-}\right)$are equal to the background levels of the flux $f(x, y)$ far enough from the grain boundary $(y=0)$, and $A$ is the area of the pickup loop.

To perform the integration the position of the macroscopic grain boundary line has to be extracted from the data on the measured flux $f(x, y)$. The grain boundary is defined as a line $y=y_{b}(x)$ of locations of the minima and maxima of the flux $f(x, y)$, the curve $y=y_{b}(x)$ is determined by analyzing a contour plot of $f(x, y)$. After the grain boundary line $y_{b}(x)$ is known, the flux $\phi(x)$ is computed by summing the fluxes $f(x, y)$ in a strip of length $y_{+}-y_{-}$or $w$ (in steps). As a final step the average background flux has to be subtracted, this results in

$$
\phi(x)=\frac{1}{2} \sum_{y=1}^{w} f(x, y)-\frac{f(x, 0)+f(x, w+1)}{4} w .
$$

The phase difference $\varphi(x)$ is found by using the relation $d \varphi / d x=C \phi(x) / \phi_{0}$, integrated from one end of the grain boundary line. To determine the constant $C$ and to calibrate the data measured by the pickup loops, the method described above is applied to several Abrikosov vortices visible in the films away from the grain boundaries. It is mentioned that $\phi(x)$ and $\varphi(x)$ are almost insensitive to small variations in the locations of the grain boundary, as the value of $\phi(x)$ is calculated as a sum of the fluxes $f(x, y)$ over a strip which by definition includes the main contributing terms.

The above procedure applied to the data series $\mathcal{A}$ and $\mathcal{B}$ results in the phase patterns $\varphi(x)$ shown in Fig. 1. These dependencies clearly exhibit several well-defined splinter vortices. A section of the phase difference $\varphi(x)$ marked by a rectangle in Fig. 1(a) describes a splinter vortex with a flux $\phi_{1}=0.37 \phi_{0}$. Its phase pattern is depicted in detail in Fig. 1(a), the flux image for this vortex is shown in the inset in Fig. 2. The graph shown in Fig. 1(b) clearly exhibits four well-defined splinter vortices. A section of the flux 


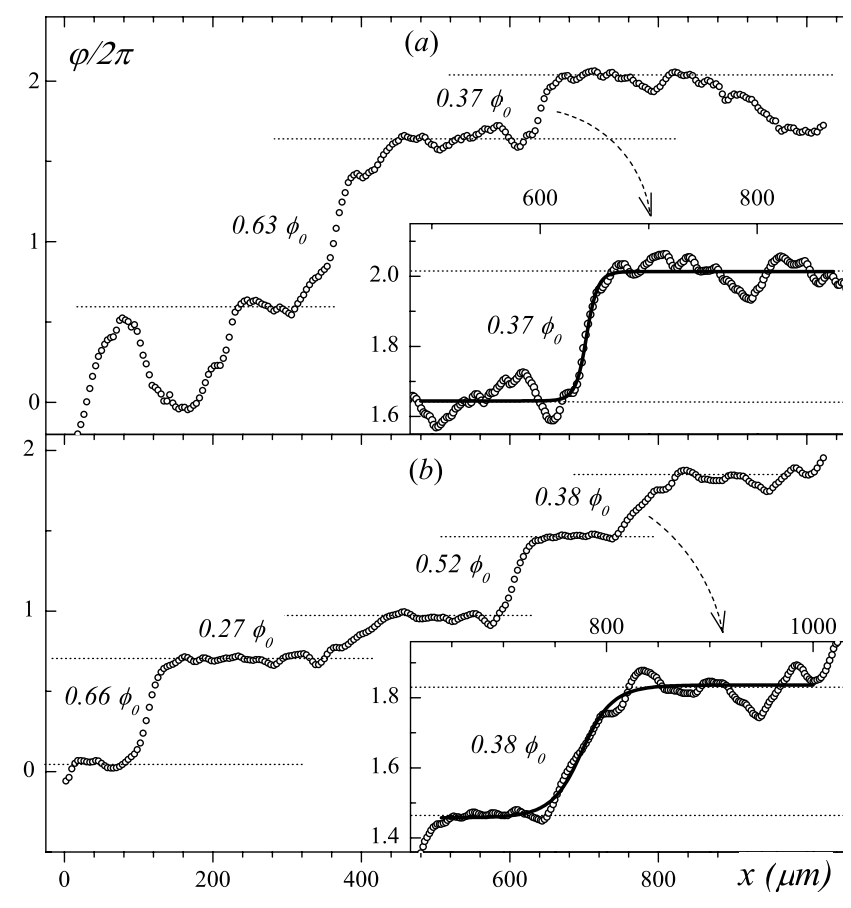

FIG. 1. The phase patterns $\varphi(x)$ for the data sets $\mathcal{A}$ (a) and $\mathcal{B}$ (b), the averages $\langle\varphi(x)\rangle$ at the splinter vortices tails are shown by dotted lines. (a) Two steps $\Delta_{1}$ and $\Delta_{2}$ allow for two splinter vortices with the complementary fluxes $\phi_{1}=0.37 \phi_{0}$ and $\phi_{2}=$ $0.63 \phi_{0}$. (b) The steps $\Delta_{1}$ and $\Delta_{2}$ vary along the grain boundary and the fluxes of the four splinter vortices do not fit to the "global" complementarity relation.

difference marked by a rectangle in Fig. 1(b) describes a splinter vortex with a flux $\phi_{1}=0.38 \phi_{0}$ and is shown in detail in the inset in Fig. 1(b).

To find the typical length scale for the splinter vortices we approximate $\varphi(x)$ by a standard vortex type solution of the sine-Gordon equation shifted by a constant

$$
\varphi_{0}(x)=4 \arctan \left[\exp \left(x / \Lambda_{0}\right)\right]+\text { const. }
$$

We use $\varphi_{a}(x)=0.37 \varphi_{0}\left(x / \Lambda_{a}\right)$ with the value of $\Lambda_{a}=$ $6.4 \mu \mathrm{m}$ to plot the solid line in the inset in Fig. 1(a). To approximate the phase pattern shown in the inset in Fig. 1(b) we use $\varphi_{b}(x)=0.38 \varphi_{0}\left(x / \Lambda_{b}\right)$ with $\Lambda_{b}=$ $12.5 \mu \mathrm{m}$.

The phase pattern $\varphi(x)$ for the data series $C$ is shown in Fig. 3, five splinter vortices and four splinter antivortices are clearly seen at the grain boundary. In the insets in Fig. 3 a splinter vortex with the flux $\phi_{\mathrm{cv}}=0.68 \phi_{0}$ and $\Lambda_{\mathrm{cv}}=$ $12.8 \mu \mathrm{m}$ and a splinter antivortex with the flux $\phi_{\mathrm{ca}}=$ $-0.37 \phi_{0}$ and $\Lambda_{\mathrm{ca}}=5.8 \mu \mathrm{m}$ are shown in detail.

The typical length of a current loop forming a splinter vortex is given by the Josephson length $\Lambda \sim \Lambda_{0}$. In our study we find that $\Lambda_{0} \sim 10 \mu \mathrm{m}$, comparing $\lambda_{\text {loc }} \sim 1 \mu \mathrm{m}$ [4] and $\Lambda_{0}$ we estimate $\left\langle j_{c}\right\rangle /\left\langle\left|j_{c}\right|\right\rangle=\left(\lambda_{\text {loc }} / \Lambda_{0}\right)^{2} \sim 10^{-2}$. A remarkable feature of the flux patterns observed for the data series $\mathcal{B}$ and $C$ is the broken "global" complementarity relation.

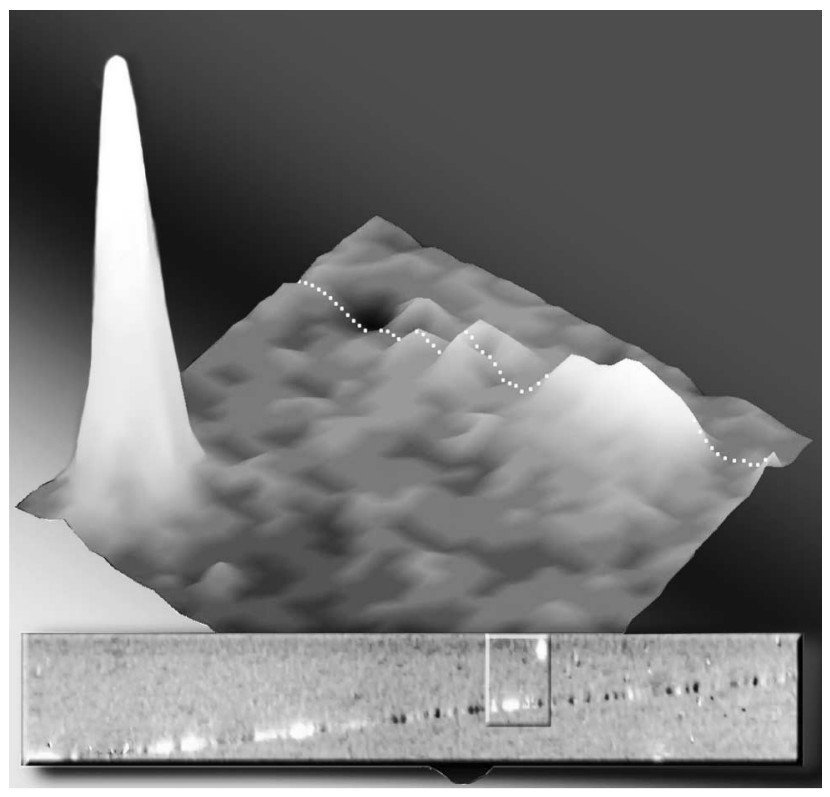

FIG. 2. Scanning SQUID microscope image of an area $60 \times$ $70 \mu \mathrm{m}^{2}$ along the grain boundary (white dotted line) in the sample $\mathcal{A}$. The splinter vortex has the flux $\phi_{1}=0.37 \phi_{0}$, its phase pattern is marked by a rectangle in Fig. 1(a). A bulk vortex is shown for comparison at the left corner away from the grain boundary. The inset at the bottom is for the flux image along the whole boundary $(1024 \mu \mathrm{m})$; the rectangle indicates the location of the splinter and bulk vortices.

Splinter vortices originate on a background of a "dense" linear array of interchanging 0 and $\pi$ junctions [18]. In this dense array the lengths $l_{0}$ and $l_{\pi}$ of the junctions are much less than the local Josephson length $\lambda_{\text {loc }}$. To understand the generation of splinter vortices qualitatively we start by considering a "dilute" array, such that $\lambda_{\mathrm{loc}} \ll l_{0}, l_{\pi}$, and arrive at a dense array by increasing the value of $\lambda_{\text {loc }}$. Indeed, consider an array of interchanging 0 and $\pi$

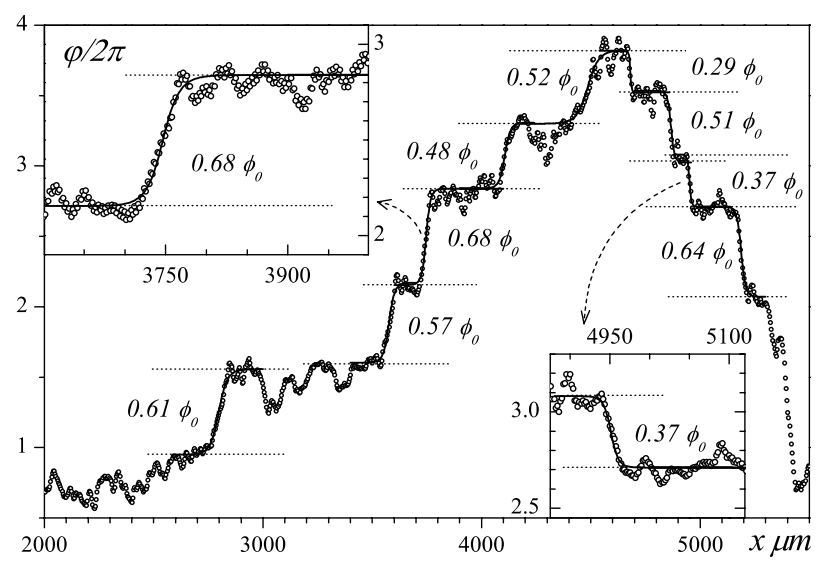

FIG. 3. The phase pattern $\varphi(x)$ for the data series $C$, the averages $\langle\varphi(x)\rangle$ at the splinter vortices tails are shown by dotted lines. The steps $\Delta_{1}$ and $\Delta_{2}$ vary along the grain boundary and the fluxes of the splinter vortices and antivortices fit to the "global" complementarity relation in two cases only.

067004-3 


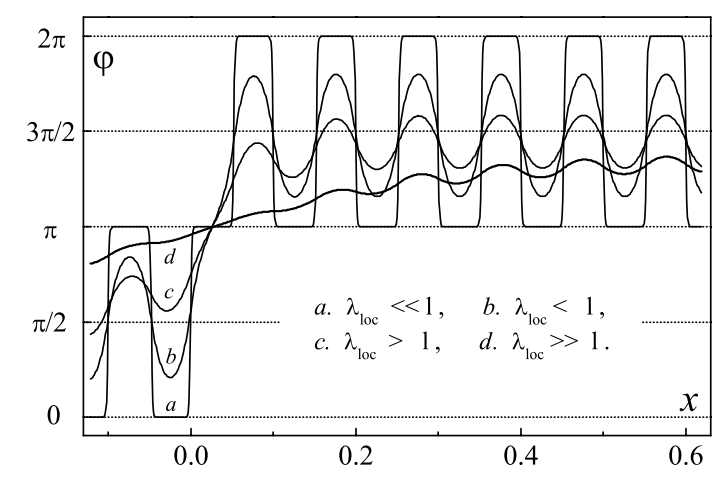

FIG. 4. Transformation of the phase pattern $\varphi(x)$ from a function generated by a row of single $\pi$ vortices and $\pi$ antivortices $(a)$ to a superposition of a smooth phase shift and a small rapidly alternating phase $(d)$.

junctions with a period $l=l_{0}+l_{\pi}, l_{0} \gtrsim l_{\pi}$, and a stepwise alternating critical current density taking the values $\pm j_{c}$ with $\left\langle j_{c}\right\rangle=j_{c}\left(l_{0}-l_{\pi}\right) / l \neq 0$.

In the equilibrium state of a dilute array of interchanging 0 and $\pi$ junctions, single $\pi$ vortices and/or $\pi$ antivortices originate at each of the $0-\pi$ connection points as shown in Fig. 4 (curve $a$ ) [20]. If a $\pi$ antivortex follows a $\pi$ vortex [as shown in Fig. 4 (curve $a$ ) for $x>0$ ] then the total flux in the system is zero. A splinter vortex develops from the phase difference $\varphi(x)$ shown in Fig. 4 (curve $a$ ). In this case the system carries flux $\phi_{0} / 2$, as an "extra" $\pi$ vortex is located at the point $x=0$.

Now increase $\lambda_{\text {loc }}$, i.e., the width of the $\pi$ vortices and $\pi$ antivortices. At a certain $\lambda_{\text {loc }}$ the "cores" of these vortices and antivortices start to overlap [21]. This overlapping results in two basic consequences. First, the average value of the phase difference noticeably shifts as the pairs of $\pi$ vortices and $\pi$ antivortices partially cancel each other. Second, the amplitude of the rapid alternations with the period $l$ drastically decreases.

The change of $\varphi(x)$ from a stepwise curve generated by a row of single $\pi$ vortices and $\pi$ antivortices $(a)$ to a superposition of a smooth and small rapidly alternating phase $(d)$ is shown in Fig. 4. The smooth phase has a length scale of order of $\Lambda$ and the alternating phase has a period of $l$. A splinter vortex with a flux $\phi_{1}=0.34 \phi_{0}$ is shown in Fig. 4 (phase $d$ ). It is seen from Fig. 4 that the flux $0.50 \phi_{0}-$ $0.34 \phi_{0}=0.16 \phi_{0}$ is steadily "pushed" out to infinity (to the edges) as the value of $\lambda_{\text {loc }}$ increases.

In summary, we have observed several sets of splinter vortices at asymmetric $45^{\circ}$ [001]-tilt grain boundaries in $\mathrm{YBa}_{2} \mathrm{Cu}_{3} \mathrm{O}_{7-\delta}$ films. The mechanism used to explain the splinter vortices has been described without adjustable parameters. It does not depend on the existence of an imaginary component to the order parameter, either intrinsic to the bulk $[22,23]$, or induced by the grain boundary interface [24-29].

This research is supported by Grant No. 2000011 from the United States -Israel Binational Science Foundation
(BSF), Jerusalem, Israel, by the BMBF (13N6918), and by the ESF pishift-programme.

[1] A. A. Abrikosov, Zh. Eksp. Teor. Fiz. 32, 1442 (1957) [Sov. Phys, JETP 5, 1174 (1957)].

[2] B. D. Josephson, Adv. Phys. 14, 419 (1965).

[3] C.C. Tsuei and J.R. Kirtley, Rev. Mod Phys. 72, 969 (2000).

[4] J. Mannhart et al., Phys. Rev. Lett. 77, 2782 (1996).

[5] Z.G. Ivanov et al., in Proceedings of the Beijing International Conference on High-Temperature Superconductivity (BHTSC '92), edited by Z. Gan (World Scientific, Singapore, 1992), p. 722.

[6] N. G. Chew et al., Appl. Phys. Lett. 60, 1516 (1992); R. G. Humphreys et al., in Proceedings of the 2nd Workshop on HTS Applications and New Materials, edited by D. H. A. Blank (University of Twente, Enschede, 1995), p. 16.

[7] C. A. Copetti et al., Physica (Amsterdam) C253, 63 (1995).

[8] H. Hilgenkamp, J. Mannhart, and B. Mayer, Phys. Rev. B 53, 14586 (1996).

[9] D. J. Van Harlingen, Rev. Mod. Phys. 67, 515 (1995).

[10] C. L. Jia et al., Physica (Amsterdam) C196, 211 (1992).

[11] S. J. Rosner, K. Char, and G. Zaharchuk, Appl. Phys. Lett. 60, 1010 (1992).

[12] C. Træholt et al., Physica (Amsterdam) C230, 425 (1994).

[13] J. W. Seo et al., Physica (Amsterdam) C245, 25 (1995).

[14] J. H. Miller et al., Phys. Rev. Lett. 74, 2347 (1995).

[15] F. P. Rogers, Master's thesis, MIT, Boston, 1983; L. N. $\mathrm{Vu}$ and D. J. van Harlingen, IEEE Trans. Appl. Supercond. 3, 1918 (1993); R. C. Black et al., Appl. Phys. Lett. 62, 2128 (1993).

[16] J.R. Kirtley et al., Appl. Phys. Lett. 66, 1138 (1995).

[17] R. G. Mints and V. G. Kogan, Phys. Rev. B 55, R8682 (1997).

[18] R. G. Mints, Phys. Rev. B 57, 3221 (1998).

[19] R. G. Mints and Ilya Papiashvili, Phys. Rev. B 64, 134501 (2001).

[20] J. H. Xu, J. H. Miller, Jr., and C. S. Ting, Phys. Rev. B 51, 11958 (1995).

[21] It is worth noting that due to the nonlinearity of the Josephson electrodynamics $\varphi(x)$ is not simply a sum of the contributions of single $\pi$ vortices (antivortices).

[22] D. B. Bailey, M. Sigrist, and R. B. Laughlin, Phys. Rev. B 55, 15239 (1997).

[23] M. Sigrist, Prog. Theor. Phys. 99, 899 (1998).

[24] M. Matsumoto and H. Shiba, J. Phys. Soc. Jpn. 64, 1703 (1995); M. Matsumoto and H. Shiba, ibid. 64, 3384 (1995);

M. Matsumoto and H. Shiba, ibid. 64, 4867 (1995).

[25] A. B. Kuklov and M. Sigrist, Int. J. Mod. Phys. B 11, 1113 (1997); A. B. Kuklov, Phys. Rev. B 52, R7002 (1995).

[26] M.E. Simon and C. M. Varma, Phys. Rev. B 60, 9744 (1999).

[27] A. V. Balatsky, Phys. Rev. Lett. 80, 1972 (1998).

[28] J.-X. Zhu and C.S. Ting, Phys. Rev. B 60, R3739 (1999).

[29] M. I. Salkola and J. R. Schrieffer, Phys. Rev. B 58, R5952 (1998). 